\title{
SOVA DECODING OF CONCATENATED CONVOLUTIONAL ENCODERS AND CPM SCHEME OVER AWGN AND FADING CHANNELS
}

\author{
Manjeet Singh - contact \\ Ian J. Wassell \\ Laboratory for Communications Engineering \\ Department of Engineering \\ Trumpington Street \\ University of Cambridge \\ Cambridge CB2 1PZ \\ Phone: +44-(0)1223-766514, Fax: $\quad+44-(0) 1223-766517$ \\ Email: ms308@eng.cam.ac.uk
}

\begin{abstract}
In this paper, Continuous Phase Modulation (CPM) is investigated. We employ the decomposed CPM model where the model is decomposed into a Continuous Phase Encoder (CPE) and a Memoryless Modulator (MM). This allows us to concentrate on the encoding aspects without interference from the modulator. By designing a convolutional channel encoder (CE), to operate over the same algebra as the CPE, the CE and the CPE can be combined and treated in a unified manner. This combination is called an extended CE, as the $C P E$ is effectively an extension of the $C E$ and is a convolutional encoder. In this research, a 1/2 rate CE over the ring of integers modulo 4 is combined with the CPE of a 4-ary CPM scheme whose modulation index is $h=1 / 4$. Using this trellis coded modulation design; the paper explains the design of a serially concatenated channel coded system, where the inner encoder is the quaternary extended CE and the outer encoder a quaternary convolutional encoder. SOVA decoding is employed. Simulations show that a BER of $2.6 \times 10^{-4}$ at $0.3 d B$ SNR is obtainable in an AWGN channel.
\end{abstract}

\section{INTRODUCTION}

Nyquist [1] and Shannon [2] provided the necessary background theory for digital communications at a time when nobody could have predicted how important it would become in the near future. Digital communications was used in World War II for military communications for reasons of secrecy and resistance to jamming, however, its commercial utilization started with the advent of space exploration in the late 1950's and the advent of solid-state technology. Using analogue to digital converters (ADC's), analogue signals could be easily transformed into discreet, quantized and binary coded values.
From then on, the digital revolution in communications has continued apace.

The basic elements of a digital point-to-point communications system are illustrated in Figure 1. The source generates analog or digital signals such as voice, video or a stream of binary digits. The transmitter converts the signal generated by the source to a suitable form to be transmitted over the waveform channel, which may be a pair of wires, coaxial cable or a radio link. The receiver attempts to retrieve the source output from the channel output and presents the results to the user, which may be the human ear, eye or a computer.

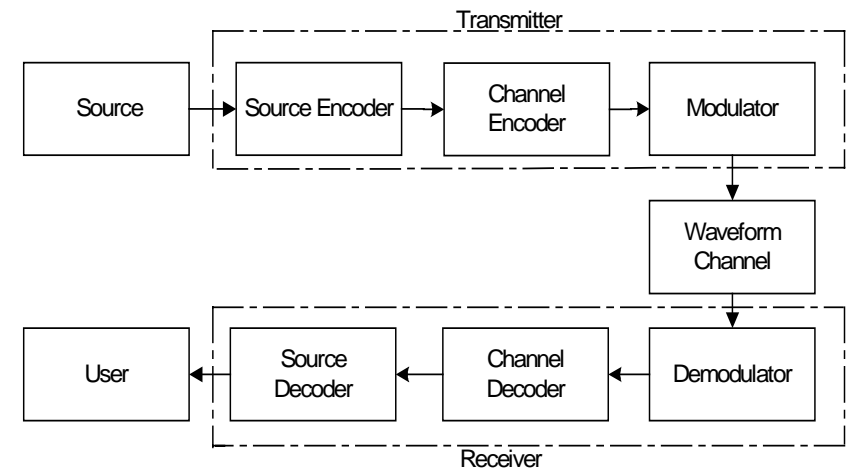

Figure 1. Block diagram of a digital point-to-point communications system.

In this paper, we are concerned with the part of the communications system between the channel encoder input and the channel decoder output. Without loss of generality, we assume that the output of the source encoder is a stream of independent and identically distributed random variables. We focus on the characteristics and design of the channel encoder, modulator and their counterparts at the receiver. 
We are particularly interested in digital modulation schemes that are both bandwidth and energy efficient. As the bandwidth is a finite resource due to physical limitations or government regulations and the demand for information transfer is increasing, efficient ways of using a given bandwidth are of great interest. One such bandwidth and energy efficient digital modulation scheme is Continuous Phase Modulation (CPM).

\section{CONTINUOUS PHASE MODULATION}

Continuous Phase Modulation (CPM) schemes belong to a class of constant-envelope digital modulation schemes that are both bandwidth and energy efficient [3, 4, 5]. This class is based on phase modulation schemes where phase continuity in the transmitted signal is maintained to shape the transmitted spectrum. The constant-envelope nature of the signals makes CPM useful also for nonlinear and /or fading channels, such as the satellite and the mobile radio channels.

Besides providing spectral economy, CPM schemes also exhibit a "coding gain" when compared to PSK modulation. This "coding gain" is due to the memory that is introduced by the phase-shaping filter and the decoder can exploit this. CPM modulation exhibits memory that resembles in many ways how a convolutionally encoded data sequence exhibits memory - in both cases, a "trellis" can be used to display the possible output signals (this is why convolutional encoders are used with CPM in this paper).

In 1988, Rimoldi, based on a theory by Massey [6], showed that a CPM scheme can be decomposed into a Continuous Phase Encoder (CPE) followed by a Memoryless Modulator (MM), where the CPE is linear modulo some integer $M$ and time invariant and the MM another time invariant device [7]. Decomposing the CPM has 2 advantages [7]. Firstly, the "encoding" operation can be studied independently of the modulation. This allows the CPE to be combined with a channel encoder and be treated as a unified channel encoder. The second advantage is that the isolation of the MM allows the cascade of the MM, the waveform channel (e.g. additive white Gaussian noise (AWGN)) and the demodulator to be modeled as a discrete memoryless channel (DMC).

The work in this paper is based on this decomposition approach to CPM. We optimally combine a convolutional coder with the CPE to create a trellis coded modulation scheme. The necessary conditions on how to combine a convolutional encoder with the CPE without the use of a mapper are given in [8]. One condition, among others, is that both the encoder and the CPE must operate over the same algebra. This is unlike the usual approach where mappers are pertinent $[3,9]$.

Here, we combine a $1 / 2$ rate 4 -ary convolutional encoder, hereafter called a channel encoder (CE), with the CPE of a 4ary CPM scheme. Such a combination is called an extended $C E$ and is effectively a convolutional encoder. This is made possible by designing both the $\mathrm{CE}$ and the CPE to operate over the ring of integers modulo 4. A serially concatenated channel coding system is designed where the outer channel encoder is a quaternary convolutional encoder and the inner encoder the quaternary extended CE. A 2 raised cosine $(2 R C)$ CPM scheme with a modulation index of $h=1 / 4$ is considered. Soft decoding techniques are investigated where the soft output information from the inner Soft-Output Viterbi Algoroithm (SOVA) is used to enhance the performance of the outer soft input Viterbi decoder.

\section{SYSTEM DESIGN}

This section explains the design of the coding system used in the study. Referring to Figure 2:

a. The outer encoder is a quaternary convolutional encoder while the inner encoder is the quaternary extended CE. As both inner and outer encoders operate over the same algebra structure, no mapper is required to link them.

b. The inner SOVA decoder outputs hard decision symbols and reliability information, the latter being used by the outer decoder to enhance its decoding capabilities. Once again, no mapper is required between the inner and outer decoders as they both operate over the same algebra structure.

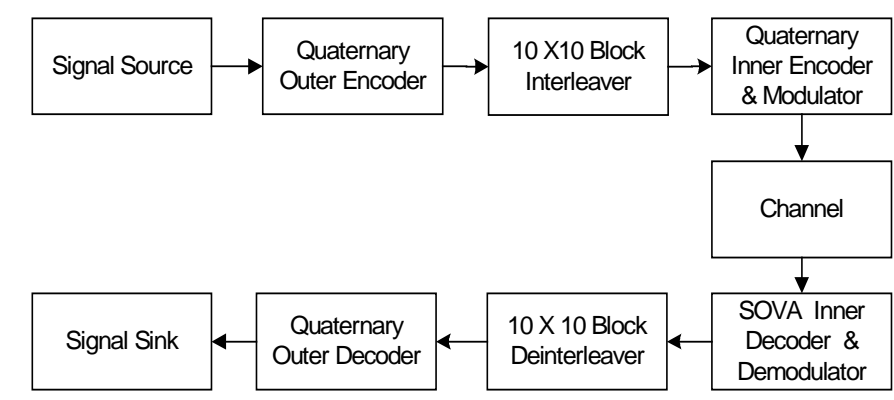

Figure 2. Block diagram of the serially concatenated channel coding system.

c. $10 \mathrm{X}$ 10-bock interleavers/ deinterleavers are used to permutate the codewords between the encoders/ decoders.

\section{A. Design of the extended CE}

Figure 3 shows the design of the extended CE. For the purpose of system evaluation, a $2 R C$ (partial-response) scheme with a modulation index $h=1 / 4(M=4)$ is used. The CPE has two memory (delay) cells. One cell stores the previous transmitted symbol while the other memory cell stores the sum $(\bmod 4)$ of all previously transmitted symbols. The phase response of the incoming signal, thus, lasts two symbol intervals $(L=2)$. This technique improves bandwidth efficiency by introducing controlled interference between symbols. 
The extended CE is a $1 / 2$ quaternary convolutional encoder. It has 3 memory cells (memory cells D1 and D2 form part of the CPE) and generates 64 states $\left(4^{3}\right)$. As there are 4 branches/ waveforms emanating from and arriving at each state and each "analog" waveform is made of 8 discrete samples, a total of 8 X 4 X $64=2048$ waveform samples would require processing for the extended CE at each iteration of the Viterbi algorithm.

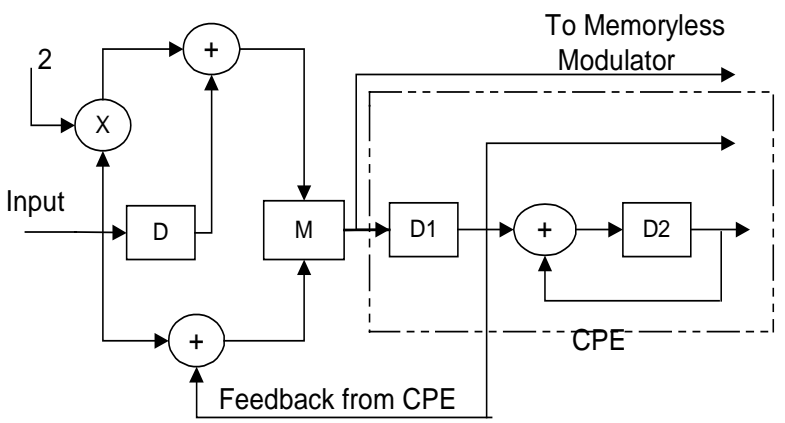

Figure 3. $1 / 2$ rate extended $C E$ with feedback from the CPE. D denotes a memory cell while $M$ denotes a multiplexer. Additions and multiplications are all modulo 4. D1 and D2 are the memory cells of the CPE. (Source: Manjeet Singh, Ian J. Wassell, "Effective Channel Coding of Serially Concatenated Encoders and CPM over AWGN and Rician Channels", MILCOM 2000 Conference Proceedings.

(C) 2000 IEEE).

By designing the $\mathrm{CE}$ to operate over the same algebra as the $\mathrm{CPE}$, no mapper is required to integrate them. This allows the state of the CPE to be fed back and be used by the CE, enabling the use of a $\mathrm{CE}$ with a shorter constraint length. Such a combination is called an extended CE, as the CPE is now an extension of the CE.

\section{B. Design of the Memoryless Modulator}

The design of the MM is shown in Figure 4 below.

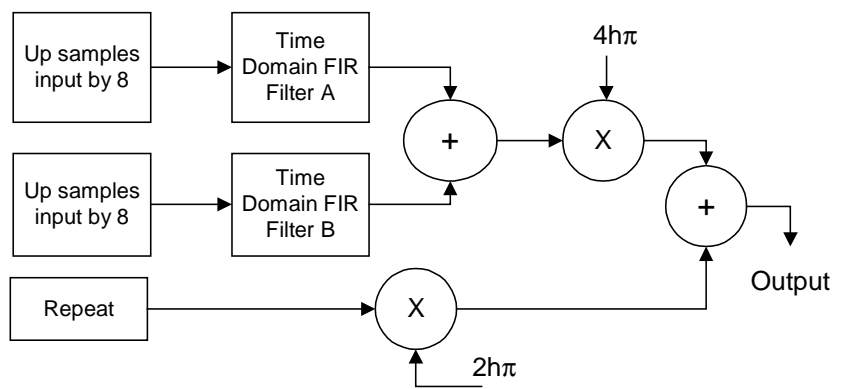

Figure 4. Block diagram of the memoryless modulator. The Up Sample and Repeat blocks increase the samples of the input signals by a factor of 8 . $h$ denotes the modulation index.

The raised cosine $(R C)$ phase shaping function is implemented using two finite impulse response (FIR) digital filters, FIR filter A and FIR filter B. In this study, each waveform is made up of eight discrete samples.

\section{Design of the Outer Encoder}

The design of the quaternary outer convolutional encoder is shown in Figure 5 below. Its design is quite similar to the extended CE shown in Figure 3.

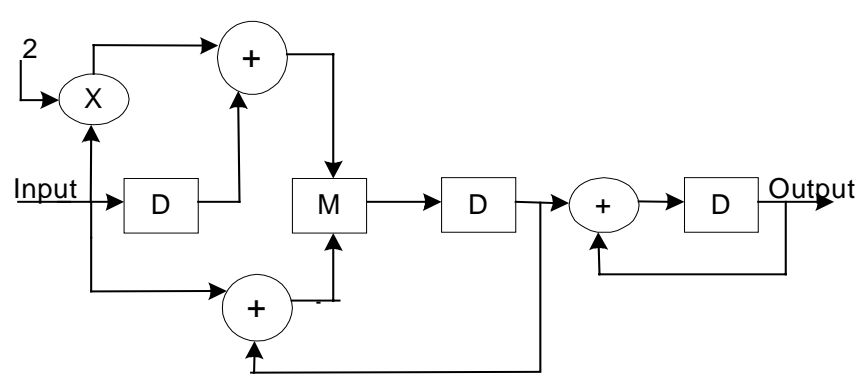

Figure 5. 1/2 rate quaternary outer encoder. D denotes a memory cell while $M$ denotes a multiplexer. Additions and multiplications are modulo 4.

\section{Design of the Viterbi Decoders}

The general overview of the Viterbi decoder is shown in Figure 6. Its design is based on the concept in [10].

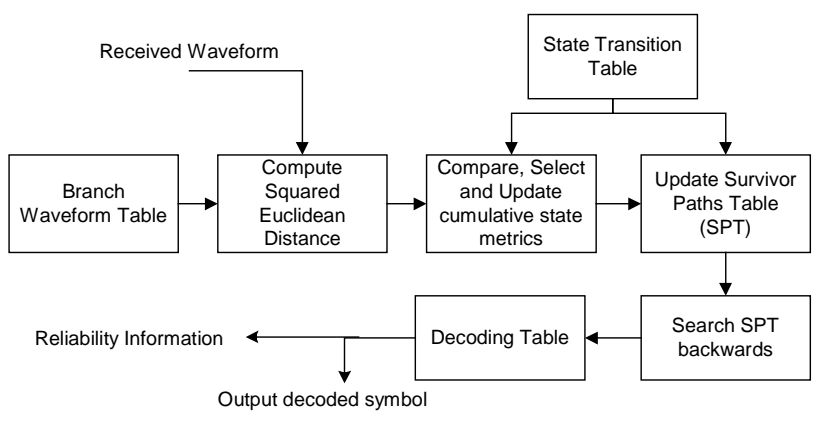

Figure 6. High-level diagram of the adaptable Viterbi Decoders

To demodulate the received signal, the following three tables were used. They were generated by the modulator and are used by the demodulator to decode the received symbol sequence. The tables are stored in ROMs in the demodulator.

a. State Transition Table - which takes as its inputs the current state and input symbol and outputs the next state.

b. Waveform Table - which takes as inputs the current state and symbol input and generates the output waveform.

c. Decoding Table - which takes as its inputs the current state and previous state and outputs the transmitted symbol. 
When a waveform is received, (corrupted by additive noise or fading), the waveform table is used to calculate the incremental squared Euclidean distance (SED) between the received waveforms and the set of all known waveforms the transmitter can transmit. These distances (or branch metrics) serve as a probability that a given waveform/ symbol was actually sent. The minimum SED corresponds to the most likely signal transmitted. These branch metrics are not used in isolation. They are used in conjunction with the probabilities (state metrics) associated with the states of the trellis that each of the branches departs from. In this way, the SED between the entire received sequence and the most likely path through the trellis can be calculated on a symbol-by-symbol basis.

Using the minimum SED, the state transition table determines the survivor path through the trellis. The decoding table back tracks through the trellis, to determine the most likely signal transmitted. It is assumed that there are no parallel transitions between states. The advantage of using these tables is that for the same value of $L$ the Viterbi decoders need not be redesigned for each new scheme to accommodate more or less states or a different symbol alphabet size. Apart from outputting the decoded symbol, the SOVA decoder also output reliability information, which is used to improve the performance of the outer decoder.

\section{SPW SIMULATIONS AND RESULTS}

The entire coding system was built in software and tested using Monte Carlo based simulations. The results obtained are in terms of the bit error rate (BER) as a function of the signal to noise ratio (SNR).

The simulations were executed in three types of channels, the additive white Gaussian noise (AWGN) channel, Rician and Rayleigh fading channels. For the simulations in the Rician fading channel, the channel was designed with a Rician parameter $(K)$ of 10 . This parameter is defined as:

$$
K=\frac{\text { power of dominant path }}{\text { power in scattered path }}
$$

When $K=0$, the channel is Rayleigh, and if $K$ is infinite, the channel is Gaussian. The fades have a high probability of being very deep when $K=0$ to being very shallow when $K=32$ (approaching Gaussian) [11].

In all the simulations, a normalized bandwidth of $B T_{s}=1.2$ was assumed where $B$ is the bandwidth and $T_{s}$, the symbol duration. For the quaternary $2 R C$ scheme, this bandwidth contains approximately $99.97 \%$ of the total power. The overall code rate of the system is $1 / 4$ (both the inner and outer encoders have a code rate of $1 / 2$ ) and each encoder has 64 states.

Results of all the simulations are shown graphically in Figures 7 (a) \& (b). Figure 7(a) shows the results obtained in the fading channels while Figure 7 (b) shows the results obtained in an AWGN channel. Using a 10 X 10 block interleaver, a BER of $2.6 \times 10^{-4}$ at $0.3 \mathrm{~dB}$ SNR was achievable in the AWGN channel.

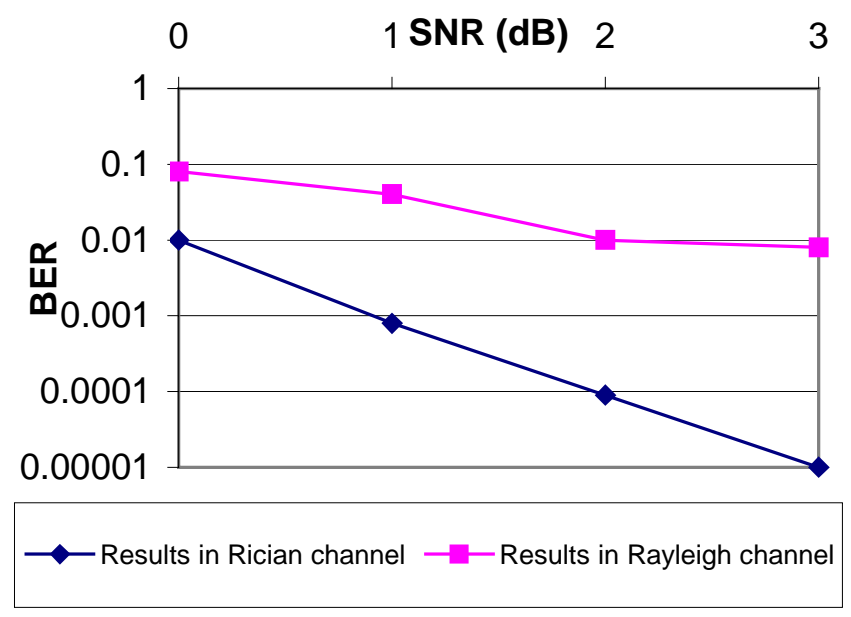

Figure 7a. BER results obtained in fading channels.

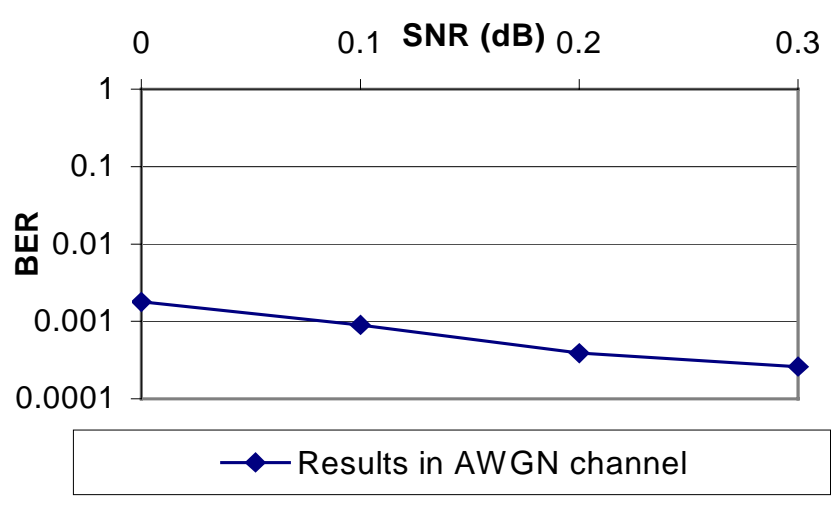

Figure $7 b$. BER results obtained in an AWGN channel.

The graph clearly shows the extremely good results that can be achieved, especially in the AWGN channel. These results are comparable to designs employing turbo coding or iterative decoding.

\section{DISCUSSION AND CONCLUSION}

A SOVA serially interleaved concatenated coding scheme has been studied. It consists of the cascade of an outer encoder, an interleaver permuting the outer codeword bits and an inner encoder whose input words are the permuted outer codewords. Soft decoding was investigated where the soft output from the SOVA inner decoder was used by the outer decoder to enhance its decoding capabilities. The scheme was tested using Monte Carlo based simulations over AWGN, Rician and Rayleigh fading channels. 
The results show good error correcting capabilities, comparable to schemes employing turbo coding. The main potential area of application of the configuration is the improvement of already established standards, for example GSM and cellular digital packet data.

\section{ACKNOWLEDGEMENTS}

The authors are grateful to AT\&T Labs (Cambridge) for the use of their equipment in the research and the Center for Wireless Communications (National University of Singapore) for their support.

\section{REFERENCES}

[1] H. Nyquist, "Certain factors affecting telegraph speed”, Bell System Technical Journal, Vol 3, No.2, pp 324346, Apr.1924.

[2] C.E. Shannon, "A mathematical theory of communication - Parts I \& II", Bell System Technical Journal, Vol.27, No.3, pp. 379-423, Jul.1948.

[3] T.Aulin, C.-E.W.Sundberg, "Continuous Phase Modulation - Part I: Full response signalling”, IEEE Trans. Commun., Vol.COM-29, pp. 196-209, Mar. 1981

[4] T.Aulin, N.Rydbeck and C.-E.W.Sundberg, "Continuous Phase Modulation - Part II: Partial response signalling”, IEEE Trans. Commun., Vol.COM-29, pp. 210225, Mar. 1981

[5] J.B. Anderson, T. Aulin and C.E. Sundberg, "Digital Phase Modulation”, New York: Plenum, 1986.

[6] J.L Massey, "The how and why of channel coding," in Proc. Int. Zurich Seminar, Mar.1984, pp. F11(67)-F17(73).

[7] B. Rimoldi, "Continuous phase modulation and coding from bandwidth and energy efficiency," $\mathrm{PhD}$ dissertation, Swiss Fed. Inst. Technol., 1988.

[8] Quinn Li, "On bandwidth and energy efficient digital modulation schemes," $\mathrm{PhD}$ dissertation, Sever Institute, University of Washington, 1996.

[9] S.V. Pizzi and S.G. Wilson, "Convolutional coding combined with Continuous Phase Modulation," IEEE Trans. Commun., vol.33, pp.20-29, Jan.1985.

[10] C.J.A. Levita, M. Benaissa and I.J. Wassell, "Adaptable Viterbi detector for decomposed CPM model over ring of integers," IEE Proc. Commun, vol 147, no.3, pp. 137143 , June 2000.

[11] R. Steele (Editor), Mobile Radio Communications, London: Pentech Press, 1994. 